\title{
PERCEPCIONES DE ALUMNOS Y PROFESORES ACERCA DE LA DIFICULTAD DE APRENDIZAJE DE ESTRUCTURAS GRAMATICALES COMPlejas En ESTUdiantes de Pedagogía en InglÉs*
}

\author{
Roxana Orrego ${ }^{* *}$, Néstor Singer ${ }^{* * *}$, Ricardo Úbeda**** ${ }^{* i x t o ~ Y a ́ n ̃ e z ~}{ }^{* \star * *}$
}

\section{Resumen}

Este estudio pretende explorar las representaciones que alumnos y profesores de la Pedagogía de Inglés de la Universidad de Santiago de Chile tienen respecto a dichas estructuras estudiadas durante el primer semestre del primer año de la carrera. En este estudio se realizó una encuesta para recabar datos de alumnos y profesores del programa señalado. Se establece que la complejidad está asociada mayoritariamente a la frase verbal. Luego, se comprueba que dichas estructuras sí poseen una carga innata de complejidad para los estudiantes. Finalmente, el estudio concluye con algunos lineamientos para el diseño de materiales y posibles estudios futuros.

Palabras clave: estructuras gramaticales, complejidad, representaciones sociales, enseñanza de inglés como lengua extranjera.

\section{LEARNERS' AND TEACHERS' BELIEFS REGARDING THE LEARNING DIFFICULTY OF COMPLEX GRAMMAR STRUCTURES IN STUDENTS OF AN ENGLISH LANGUAGE TEACHING UNDERGRADUATE PROGRAMME}

\begin{abstract}
This study aims to explore the social representations held by the learners and teachers of the undergraduate English language teaching programme at the Universidad de Santiago de Chile, specifically the grammar structures taught during the first semester of the first year. For this research, a survey to collect the data from students and teachers was carried out. It is thought that complexity is associated to the verb phrase. Later, it was confirmed that these structures do have an innate charge of complexity for learners. This study concludes with some guidelines for the design of materials and future possible research.
\end{abstract}

Keywords: grammar structures, complexity, social representations, teaching English as a foreign language.

* Investigación adscrita al proyecto DICYT 031651OR para 2016-2018 denominado El impacto de la retroalimentación interaccional correctiva conocida como 'recast' en el aprendizaje de estructuras gramaticales en lengua inglesa en discurso oral.

** Chilena, Doctora en Lingüística por la Pontificia Universidad Católica de Valparaíso, Académica de la Universidad de Santiago de Chile, Santiago, Chile. roxana.orrego@usach.cl

*** Chileno. MA TESOL por The University of Manchester. Académico de la Universidad de Santiago de Chile, Santiago, Chile. nestor.singer@usach.cl

**** Chileno. Magíster en Lingüística Inglesa por la Universidad de Chile. Académico Universidad de Santiago de Chile, Santiago, Chile. ricardo.ubeda@usach.cl

***** Chileno. Doctor en Educación y Sociedad por la Universidad Autónoma de Barcelona, Académico de la Universidad de Santiago de Chile, Santiago, Chile.sixto.yanez@usach.cl 


\section{Introducción}

Durante muchos años, la gramática fue el centro en la enseñanza del inglés como idioma extranjero. Sin embargo, con la aparición del Enfoque Comunicativo (Hymes, 1972; Savignon, 1983) durante la década del 70, esta concepción cambió radicalmente, dando prioridad a la competencia comunicativa. Dentro de este enfoque, la gramática fue considerada superficialmente y como un mero medio para comunicarse en una segunda lengua (L2). No obstante, en las postrimerías del siglo XXI, la eficacia del enfoque comunicativo en cuanto al desarrollo de la competencia comunicativa ha sido cuestionado (Widdowson, 1998; Cook, 2008) y nuevos enfoques centrados en la atención a la forma y al significado han surgido con el objetivo de desarrollar una competencia comunicativa efectiva que permita al aprendiente de una L2 desenvolverse fluidamente y con precisión gramatical en diversos contextos comunicativos (Hall, 2011).

En dicho contexto, esta investigación surge de la necesidad de explorar las representaciones de los estudiantes y los profesores en relación a la dificultad cognitiva asociada a las estructuras gramaticales del idioma inglés durante el primer semestre del programa de Pedagogía en Inglés de la Universidad de Santiago de Chile (USACH), el que sigue un enfoque comunicativo. Esto tiene como propósito 1) generar conciencia de la complejidad lingüística como fenómeno cognitivo natural que experimentan los alumnos y 2) potenciar metodologías, estrategias didácticas e instancias de aprendizaje que permitan al profesor facilitar los procesos de enseñanza aprendizaje de inglés como lengua extranjera. Tras un análisis de la literatura reciente que aborda estas temáticas, no se logró recabar estudios en torno a estas percepciones en Chile. Por tal razón, se establecen las siguientes preguntas de investigación:

1. ¿Qué estructuras gramaticales del primer año son percibidas como complejas por estudiantes y profesores chilenos del programa de Pedagogía en inglés en la Universidad de Santiago de Chile?

2. ¿Dónde radica la complejidad de las estructuras identificadas por los alumnos y profesores? ¿En la configuración de la estructura en sí, en el costo de procesamiento de la estructura o la combinación de ambos? 
En los siguientes apartados se expondrán brevemente los sustentos teóricos que contribuyen a este estudio, así como la metodología de trabajo y elaboración del instrumento de recolección de datos.

\section{Marco Teórico}

Esta sección aborda los principios subyacentes del estudio; la teoría de procesamiento (Pienemann, 2005), así como los principios de procesamiento del input lingüístico. Posteriormente, ahonda en la noción de complejidad y sus características como parte del universo individual cognitivo de los alumnos. Este apartado concluye con una síntesis del origen de las representaciones sociales y su potencial rol en la construcción de la noción de complejidad.

\subsection{La Teoría de Procesamiento}

La Teoría de Procesamiento (Pienemann, 2005; Keatinge y Kebler, 2009) ofrece una perspectiva psicolingüística que da cuenta de las etapas y secuencias que constituyen el proceso de aprendizaje de los diferentes subsistemas de una lengua extranjera, y del aprendizaje de las relaciones de forma y significado(s) de las diferentes estructuras morfosintácticas del sistema. Jordan (2004) argumenta que este modelo se distingue por permitir hacer predicciones explícitas sobre (a) relaciones morfosintácticas, (b) orden y secuencias de aprendizaje, y (c) la validez inter lingüística del proceso.

El principio fundamental de este modelo teórico es que el aprendizaje de una lengua está determinado por la estructura cognitiva del procesamiento lingüístico. En este sentido, Pienemann (2011) explica que el aprendizaje de formas y funciones lingüísticas de una L2 esta 'restringido' por la naturaleza de la forma que un individuo puede procesar efectivamente durante las diferentes etapas del proceso de desarrollo lingüístico; de este modo, los individuos solo pueden aprender aquellas formas y funciones lingüísticas que pueden procesar. En la Teoría de Procesamiento se define el concepto de "procesamiento gramatical productivo» en base al principio de unificación de rasgos, cuya efectividad está determinada por el almacenamiento de la información en la memoria gramatical y su activación durante el proceso de producción lingüística. Según Buyl y Housen (2015), este proceso se realiza a través de los siguientes procedimientos de procesamiento y en una secuencia 
correlativa que no incluye necesariamente todas las etapas:

a) Los elementos léxicos son activados a través del procedimiento de procesamiento léxico.

b) La información categórica asociada a un elemento léxico es activada a través del procedimiento de procesamiento categórico.

c) A través de la unificación de la información semántica entre los constituyentes internos de una frase, la estructura lingüística a nivel de frase es activada a través del procedimiento de procesamiento de frases.

d) El procedimiento de procesamiento oracional analiza la información inter frases y activa la información sintáctica relacionada con el orden lógico de estas para la configuración sintáctico-semántica de la oración.

e) El procedimiento de procesamiento de subordinación opera sobre estructuras subordinadas activando el procesamiento productivo de las relaciones jerárquicas de dichas construcciones.

La Teoría de Procesamiento sostiene que la secuencia de etapas de aprendizaje de una L2, al igual que su activación durante el proceso de producción lingüística, están determinadas por el mismo orden en que están establecidos los procedimientos de procesamiento descritos anteriormente, determinando, de igual modo, una secuencia de procesamiento jerárquico que permite hacer predicciones explícitas sobre el orden de las etapas de aprendizaje de una L2 (Buyl y Housen, 2015).

\subsection{Procesamiento de Input lingüístico}

El proceso de aprendizaje de una L2 es complejo en cuanto resulta del procesamiento de una serie de sub-sistemas lingüísticos interrelacionados compuestos de elementos léxicos, de un sistema sintáctico abstracto, de un sistema fonológico y de normas de uso pragmático. Esta visión permite concluir que este procesamiento debe entenderse como una serie de procesos y sub procesos a nivel receptivo y productivo secuenciados en etapas de desarrollo determinadas por las capacidades cognitivas de un individuo durante el proceso de aprendizaje de una L2.

A partir de estos planteamientos teóricos, y basado en el concepto de Procesamiento de Input presentado por Chaudron (1985), VanPatten 
(2004) explica que el procesamiento gramatical debe ser entendido como el proceso a través del cual se establece una conexión directa entre una forma y su(s) significado(s). En este sentido, el autor argumenta que un modelo de procesamiento de input debe ser entendido principalmente en base a los siguientes principios:

a) Un modelo de procesamiento de input no equivale, en ninguno de sus planteamientos, a un modelo de adquisición debido a que los procesos propios de la adquisición en general, y de una de una segunda lengua en particular (reseteo de parámetros, regularización de formas y estructuras) difieren desde una perspectiva cognitiva.

b) Un modelo de procesamiento de input debe dar cuenta de las condiciones psicolingüísticas que facilitan el establecimiento de una conexión entre una forma en el input lingüístico y su(s) significado(s), además de las facilitadoras del proceso de adquisición de una L2 en general. (p. 5-6).

De acuerdo con estos planteamientos teóricos, es pertinente entender el concepto de "procesamiento» como el establecimiento de una conexión entre una forma lingüística y su(s) significado(s), teniendo como condicionante la capacidad cognitiva de un individuo de notar o percibir una forma en el input y establecer una conexión absoluta de esta con su(s) significado(s) (VanPatten, 2004). En este contexto, y desde una perspectiva cognitiva, la capacidad de procesamiento de una forma no debe ser confundida con la capacidad cognitiva de percepción o atención de esta. En este sentido, los procesos de percepción y de atención a una forma corresponden al registro acústico y a la atención consciente a esta respectivamente. Es pertinente señalar, que ambos procesos son previos a la capacidad de un individuo de asociar una forma y su(s) significado(s) particular(es), por lo que una forma puede ser percibida pero no procesada y, como consecuencia, no adquirida.

Un concepto relevante que surge de este análisis es el de intake, el cual se refiere a la parte del input que es procesada en la memoria de corto plazo, y que queda disponible para su procesamiento posterior en tareas de producción y comprensión (Krashen, 1981; Gass, 1997). En forma paralela, esta información procesada puede eventualmente pasar a formar parte del sistema de inter lengua de un individuo. 


\subsection{Principios de procesamiento de input lingüístico}

A partir de la propuesta teórica formulada por VanPatten (2004), es posible caracterizar el procesamiento de input lingüístico en base a la naturaleza propia del acto de interacción lingüística, identificando tres rasgos distintivos durante este proceso:

a) El enfoque de un individuo está en la extracción de significado del input,

b) Un individuo debe atender a una forma en el input como primera condición para el aprendizaje,

c) El proceso de atención a la forma está condicionado por las capacidades limitadas de almacenamiento y procesamiento de información.

Así, el modelo teórico de procesamiento de input da cuenta de una serie de principios que permiten explicar las etapas y secuencias de este proceso cognitivo, tal como señala la Tabla 1.

Tabla 1. Principios de Procesamiento de Input (VanPatten, 2004)

\begin{tabular}{|l|}
$\begin{array}{l}\text { Principio 1. Los individuos procesan el input para acceder al significado antes que la } \\
\text { forma. }\end{array}$ \\
\hline Principio 1a. Los individuos procesan palabras de contenido en el input lingüístico. \\
\hline $\begin{array}{l}\text { Principio 1b. Los individuos atienden a elementos léxicos en vez de formas } \\
\text { gramaticales para extraer significado cuando ambas unidades expresan el mismo } \\
\text { contenido semántico. }\end{array}$ \\
\hline $\begin{array}{l}\text { Principio 1c. Los individuos procesan elementos morfológicos más significativos antes } \\
\text { que aquellos menos significativos. }\end{array}$ \\
\hline $\begin{array}{l}\text { Principio 1d. El principio de preferencia de la no-redundancia. Los individuos procesan } \\
\text { formas gramaticales significativas no redundantes antes que las formas significativas } \\
\text { redundantes. }\end{array}$ \\
\hline $\begin{array}{l}\text { Principio 1e. El principio del significado sobre la ausencia de significado. Los } \\
\text { individuos tienden a procesar formas gramaticales significativas antes que aquellas no } \\
\text { significativas en forma independiente del grado de redundancia de estas. }\end{array}$ \\
\hline $\begin{array}{l}\text { Principio 1f. Principio de disponibilidad de recursos. Para que un individuo procese } \\
\text { formas gramaticales redundantes o formas no significativas, el procesamiento } \\
\text { de información a nivel oracional no debe mermar los recursos de procesamiento } \\
\text { disponibles. }\end{array}$ \\
\hline $\begin{array}{l}\text { Principio 1g. Principio de locación oracional. A nivel oracional, un individuo procesa } \\
\text { las formas en posición inicial antes que las formas en posición final. Estas últimas, a la } \\
\text { vez, son procesadas antes que las formas en posición central. }\end{array}$
\end{tabular}

Principio 2. El principio de la primera forma nominal. Los individuos procesan la primera forma nominal en una oración como sujeto/agente. 
Principio 2a. El principio semántico-léxico. Los individuos pueden basar su procesamiento oracional en aspectos semántico-léxicos en lugar del análisis de patrones sintácticos canónicos.

Principio $2 \mathrm{~b}$. El principio de probabilidades de ocurrencia de un evento. Los individuos pueden basar su procesamiento oracional en el análisis de probabilidades de ocurrencia de un evento en lugar del análisis de patrones sintácticos canónicos.

Principio 2c. El principio de contextualización. Los individuos pueden establecer una relación de independencia del principio de la primera forma nominal si el contexto lingüístico condiciona la interpretación del significado de una oración.

\subsection{La noción de complejidad}

La noción de complejidad ha sido ampliamente estudiada como un proceso de clasificación de fenómenos lingüísticos de acuerdo a su grado de complejidad. En este contexto, Miestamo (2008) identifica dos aproximaciones complementarias al concepto de complejidad: la perspectiva objetiva o absoluta y la perspectiva subjetiva o relativa (user-oriented). La primera, define el concepto como una propiedad característica del sistema lingüístico. La segunda, explica el concepto desde la perspectiva del costo o dificultad que experimenta el usuario del sistema lingüístico. Una alternativa para describir el concepto de complejidad lo constituye el "principio de una forma un significado» (one-form-one-meaning principle), el cual implica que un dominio lingüístico en el que se establece una relación directa entre una forma y un significado es menos complejo que un dominio en el que esta relación es múltiple.

\subsubsection{La complejidad lingüística}

El concepto de complejidad lingüística es un fenómeno inherente a los diferentes componentes del sistema lingüístico: sistema fonológico, sistema morfológico y sistema sintáctico (Kusters, 2003). Esto permite describir y definir los rasgos lingüísticos de naturaleza compleja en los diversos dominios y dar cuenta de los factores que determinan su grado de complejidad.

a) Complejidad gramatical: Un sistema gramatical es más complejo si este contiene un mayor número de expresiones gramaticalizadas para expresar distinciones semántico-pragmáticas específicas (Szmrecsanyi y Kortmann, 2009). 
b) Complejidad sintáctica: Los sistemas lingüísticos que ofrecen un mayor rango de patrones sintácticos pueden ser considerados como más difíciles de procesar en tareas de comprensión y producción.

c) Complejidad morfológica: Los sistemas lingüísticos son sistemas de inflexión más desarrollados y son más complejos en cuanto estos fenómenos son considerados como un factor de complejización debido a que estos 1) condicionan el procesamiento de una determinada forma y 2) introducen patrones de irregularidad en un sistema lingüístico.

Adicionalmente, Davydova (2011) propone el siguiente esquema de criterios para la clasificación de formas lingüísticas de acuerdo a su nivel de complejidad.

a) Configuración estructural de la forma lingüística.

b) Relaciones temporales expresadas por la forma lingüística.

c) Composición semántica de la forma lingüística.

d) Relaciones polisémicas de la forma lingüística.

e) Orden de adquisición de la forma lingüística.

f) Frecuencia de uso de la forma lingüística.

Podemos concluir que las estructuras que se presentan a los alumnos tienen una carga inherente de complejidad que puede jugar un rol crucial en el procesamiento y posterior comprensión de la estructura en cuanto a su forma y posibles significados. No obstante, dichos criterios dan cuenta de la complejidad desde un punto de vista cognitivo individual. Sin embargo, como señala Hall (2011), el proceso de aprendizaje de una lengua no es meramente un proceso mental independiente, sino más bien un proceso de interacción social en un contexto determinado. La interacción de los alumnos con los diferentes agentes del aula genera una serie de variables que pueden condicionar el aprendizaje. Desde esta perspectiva, la complejidad resulta más bien una impresión que el alumno construye en base a su vivencia en el aula, lo que conjugado con otros factores individuales, da origen a las representaciones en torno a una estructura gramatical. 


\section{Representaciones sociales}

La representación de complejidad puede ser abordada también desde una perspectiva socio-cultural si se considera al individuo y su interacción con la realidad. Por ende, es relevante el proceso de constitución de las representaciones en torno a un determinado fenómeno lingüístico.

Kornblit (2007) estipula que las relaciones «corresponden a actos del pensamiento en los cuales un sujeto se relaciona con un objeto» (p. 92) con el fin de generar conocimiento y entendimiento del mundo. Existen tres principios clave que subyacen la teoría de las representaciones: 1) el conocimiento es resultado de la interacción inmanente que existente entre los objetos sociales, 2) dicho conocimiento es generado mediante la comunicación entre individuos y 3) esta interacción perfila al lenguaje y la comunicación como los mecanismos mediante los cuales se transmite y crea la realidad (Moscovici, 1961).

De esta forma, los objetos se transforman en representaciones mediante los procesos de objetivación y el anclaje. El primero corresponde a la formación de conocimiento mediante 1) la filtración de diferentes elementos teóricos, 2) la organización de aquellos que le son útiles, y 3) el establecimiento de dicho supuesto como válido. Por su parte, el anclaje se relaciona con la forma en que este nuevo conocimiento se integra con la visión de mundo del individuo y sus representaciones previas.

Adicionalmente, Abric (1976) sugiere que las representaciones funcionan en torno a núcleos centrales y elementos periféricos. El núcleo corresponde a aquellos elementos innegociables de los que subyacen la rigidez, estabilidad y consenso de una representación. Dichos elementos corresponden a la visión de mundo del individuo; por ejemplo, opiniones, creencias y valores asociados a la memoria colectiva social. Los elementos periféricos contienen la carga flexible, de movilidad y dan cuenta de las diferencias entre individuos, ya que permiten la integración de experiencias e historias individuales.

En relación al aprendizaje de lenguas, las representaciones forman un componente fundamental que los alumnos traen al aula; por ejemplo, el rol del profesor, cómo corregir un error, el lugar que la gramática debe tener en la sala de clases, entre otros (Hall, 2011). Se puede inferir que en el proceso de construcción de significado en el aula se suscitan dos fenómenos: la generación de la competencia lingüística y las representaciones asociadas 
a dicho proceso. En otras palabras, la combinación entre la experiencia presente en el aula de los alumnos durante su proceso de aprendizaje y la interacción con sus representaciones y conocimiento previo podrían ser un factor que genera la representación de complejidad.

En consecuencia, la representación de complejidad lingüística no solo obedece a la carga inherente de una estructura, sino a un proceso socio-cultural mayor contextualizado. Este entendimiento del proceso ayudó al diseño del instrumento y la recolección de datos descritos en el siguiente apartado.

\section{Metodología}

Este estudio de caso presenta un enfoque cuali-cuantitativo no experimental y se desarrolló en base a la información obtenida de la administración de una encuesta a alumnos y profesores del programa antes señalado. Como esta investigación trata una temática escasamente abordada en la realidad nacional, se puede afirmar que es de carácter exploratorio (Hernández et al. 2010), así como también de tipo descriptivo, ya que se centra en describir las representaciones de la población consultada.

\subsection{Participantes}

La muestra estuvo constituida por 149 estudiantes y 6 profesores de lengua inglesa de la carrera de Pedagogía en Inglés de la Universidad de Santiago de Chile.

Los alumnos de Pedagogía en Inglés cursan un programa con un total de 8 asignaturas consecutivas de idioma inglés, cuyo objetivo es alcanzar el C1 según el MCER (Marco Común de Referencia Europeo) (Council of Europe, 2001). Los alumnos provienen de una serie de contextos socioculturales, por lo que la conducta de entrada al programa en cuanto al idioma inglés es diversa.

Los profesores que imparten las asignaturas de lengua inglesa son profesionales con licenciatura en inglés y título de profesor en inglés. La experiencia docente del equipo fluctúa entre 3 y más de 40 años y solo los docentes con más años de experiencia han participado en la formación de profesores. Por otra parte, algunos académicos poseen una Licenciatura, mientras otros docentes poseen el grado de Magíster y / o Doctorado. 


\subsection{Población, muestra y administración}

Como se aprecia en la Tabla 2, en este estudio se consideró la población constituida por la totalidad de estudiantes de $2^{\circ}, 3^{\circ}, 4^{\circ}$ y $5^{\circ}$ año de la carrera, y los profesores de lengua inglesa del programa. El cuestionario se aplicó a una muestra de tipo no probabilística, correspondiente a la cantidad de estudiantes que asistieron a clases en el horario en que esta se administró a los diferentes cursos y niveles y a los profesores que dictan las asignaturas de lengua inglesa.

Tabla 2. Cantidad de profesores y estudiantes separados por sexo y nivel

\begin{tabular}{|l|c|c|c|c|c|c|}
\hline \multirow{2}{*}{ Nivel } & \multicolumn{2}{|c|}{ Estudiantes } & \multirow{2}{*}{ Total } & \multicolumn{2}{|c|}{ Profesores } & \multirow{2}{*}{ Total } \\
\cline { 2 - 3 } & Hombres & Mujeres & & Hombres & Mujeres & \\
\hline $2^{\circ}$ Año & 12 & 35 & 47 & & & \\
\cline { 1 - 3 } $3^{\circ}$ Año & 06 & 35 & $\mathbf{4 1}$ & & & \\
\hline $4^{\circ}$ Año & 12 & 31 & 43 & 2 & 4 & 6 \\
\cline { 1 - 3 } $5^{\circ}$ Año & 05 & 13 & $\mathbf{1 8}$ & & & \\
\hline Total & 35 & 114 & $\mathbf{1 4 9}$ & & & \\
\hline
\end{tabular}

Los datos analizados presentan un $95 \%$ de confianza con un margen de error del 5\% y, si se considera que el universo era de 188 estudiantes y 10 profesores, se puede afirmar que los resultados de la muestra son generalizables por ser representativa y significativa según los criterios para el cálculo de muestras en Hernández et al. (2010).

\subsection{Instrumento de recolección de datos}

Las encuestas, diseñadas para determinar las percepciones de estudiantes y profesores, consideró reactivos orientados a obtener información, opiniones, y evaluaciones que permitieran concluir con objetividad y validez los hallazgos.

El instrumento, como se aprecia en la Tabla 3, se estructuró sobre una dimensión y dos sub dimensiones y tenía por propósito indagar las percepciones respecto a las estructuras gramaticales estudiadas durante el primer semestre del primer año de la carrera en ambas muestras. La encuesta se focalizó exclusivamente en los contenidos del programa del primer semestre del programa de Pedagogía en Inglés. 
Tabla 3. Matriz de elaboración de cuestionarios

\begin{tabular}{|c|c|c|c|}
\hline Dimensión & Sub dimensiones & Descriptores & Tipo reactivo \\
\hline \multirow{2}{*}{$\begin{array}{c}\text { Grado de dificultad } \\
\text { de estructura }\end{array}$} & $\begin{array}{c}\text { Estructuras } \\
\text { complejas }\end{array}$ & Listar estructuras & Selección múltiple \\
\cline { 2 - 4 } & Causas & $\begin{array}{c}\text { Jerarquizar en } \\
\text { listado }\end{array}$ & Escala ordinal \\
\hline
\end{tabular}

\subsection{Procedimiento analítico}

El análisis estadístico se realizó sobre la dimensión establecida, esto es, estructuras gramaticales complejas, determinando la frecuencia y porcentajes de cada una de estas estructuras. Para esto se utilizó el software estadístico SPSS (Statistic Package for the Social Sciences).

\section{Presentación y discusión de los resultados}

Una vez obtenidos y tabulados los resultados de la encuesta, se procedió a registrar los datos cualitativos y cuantitativos para visualizar qué tipo de estructuras gramaticales del programa primer año son percibidas como complejas e inferir si la complejidad de las estructuras identificadas radica en su configuración o en el costo de procesamiento de estas.

\subsection{Presentación de los resultados}

En las tablas a continuación presentan los resultados obtenidos de las encuestas. La Tabla 4 expresa en términos porcentuales las percepciones sobre la identificación de las estructuras complejas del idioma inglés en el primer año de estudio en la carrera, mientras que la Tabla 5 identifica las estructuras percibidas espontáneamente como complejas.

Tabla 4. Grado de dificultad asignado a estructuras gramaticales por nivel y encuestados

\begin{tabular}{|l|c|c|c|c|c|c|c|c|c|c|}
\hline \multicolumn{10}{|c|}{ Grado de dificultad (1: muy fácil / 5: muy difícil) } \\
\hline Nivel & $\begin{array}{c}\text { Presente } \\
\text { Simple }\end{array}$ & $\begin{array}{c}\text { Presente } \\
\text { Contin. }\end{array}$ & $\begin{array}{l}\text { Pasado } \\
\text { Simple }\end{array}$ & $\begin{array}{c}\text { Presente } \\
\text { Perfecto }\end{array}$ & $\begin{array}{c}\text { Comp. } \\
\text { Super. }\end{array}$ & $\begin{array}{c}\text { Contables } \\
\text { Incontables }\end{array}$ & Prepos. & $\begin{array}{c}\text { Will/ } \\
\text { Going to }\end{array}$ & Modal & $\begin{array}{c}\text { Voz } \\
\text { Pasiva }\end{array}$ \\
\hline $2^{\circ}$ Año & 1,49 & 1,98 & 1,83 & 3,02 & 2,32 & 2,68 & 3,21 & 2,70 & 2,91 & 3,21 \\
\hline $3^{\circ}$ año & 1,34 & 1,68 & 1,63 & 3,22 & 2,33 & 3,07 & 3,95 & 2,61 & 2,93 & 4,07 \\
\hline $4^{\circ}$ Año & 1,60 & 1,74 & 1,88 & 3,19 & 2,40 & 3,02 & 3,60 & 2,65 & 3,02 & 3,65 \\
\hline
\end{tabular}




\begin{tabular}{|l|l|l|l|l|l|l|l|l|l|l|}
\hline $5^{\circ}$ Año & 1,55 & 1,72 & 1,88 & 2,88 & 2,77 & 3,11 & 4,00 & 2,88 & 2,94 & 3,50 \\
\hline Promedio & 1,45 & 1,78 & 1,81 & 3,08 & 2,45 & 2,97 & 3,69 & 2,71 & 2,95 & 3,60 \\
\hline Profesores & 3,67 & 1,83 & 3,17 & 3,83 & 3,00 & 3,17 & 4,50 & 2,00 & 3,67 & 3,83 \\
\hline $\begin{array}{l}\text { Promedio } \\
\text { alumnos y } \\
\text { profesores }\end{array}$ & 1,93 & 1,79 & 2,08 & 3,23 & 2,56 & 3,01 & 3,85 & 2,57 & 3,09 & 3,65 \\
\hline
\end{tabular}

Los resultados muestran que, en opinión de profesores y estudiantes, las cinco estructuras más complejas son el uso de preposiciones $(3,85)$, seguido de la voz pasiva $(3,65)$, el tiempo gramatical presente perfecto $(3,23)$, verbos modales $(3,09)$ y sustantivos contables e incontables $(3,01)$.

Por otra parte, la Tabla 5 muestra la frecuencia de espontaneidad con la que alumnos y profesores señalan que una estructura es compleja. Las estructuras en la lista corresponden a todas aquellas con un promedio grupal superior al 15\% y con una aparición al menos en dos de los niveles de alumnos.

Tabla 5. Frecuencia de espontaneidad de estructuras percibidas como complejas

\begin{tabular}{|l|c|c|}
\hline \multirow{2}{*}{\multicolumn{1}{|c|}{ Estructura }} & \multicolumn{2}{|c|}{ Promedio } \\
\cline { 2 - 3 } & Alumnos & Profesores \\
\hline Presente Perfecto & $41,4 \%$ & $50,0 \%$ \\
\hline Pasado Perfecto & $34,4 \%$ & - \\
\hline Voz Pasiva & $32,0 \%$ & $50,0 \%$ \\
\hline Condicionales & $24,4 \%$ & $50,0 \%$ \\
\hline Presente Perfecto Continuo & $19,2 \%$ & - \\
\hline Preposiciones & $18,4 \%$ & - \\
\hline
\end{tabular}

Al contrastar estos datos, podemos ver que existe una coherencia entre la complejidad asignada y la frecuencia entre los grupos entrevistados. No obstante, las preposiciones aparecen con menor frecuencia en relación a la dificultad cognitiva que presenta para los alumnos. De manera similar, los verbos modales que figuran en la Tabla 5 , solo quedan con un promedio de frecuencia del $11,2 \%$. Esto implica que una estructura puede ser compleja sin que se le reconozca espontáneamente como tal, pues solo al ser consultados específicamente por esta, el indicador aumenta. Lo anterior, sumado a las impresiones de los profesores, parece indicar que la dificultad está focalizada en la frase verbal en lo que respecta a la 
expresión de temporalidad, aspectualidad, modalidad y voz, mientras que las preposiciones emergen como un foco de conflicto sintáctico a nivel interoracional.

\subsection{Discusión y análisis de los resultados}

Este análisis se centrará en las tres estructuras más difíciles de procesamiento en términos cognitivos de la frase verbal, es decir, presente perfecto, la voz pasiva y el uso de preposiciones Este apartado tiene como objetivo caracterizar el grado de complejidad de dichas estructuras a partir de la propuesta teórica formulada por VanPatten (2004), que caracteriza el procesamiento de input lingüístico como un proceso de interpretación de significado basado en atención a una forma específica y condicionado por las capacidades limitadas de almacenamiento y procesamiento de información, y al esquema de criterios para la clasificación de la complejidad de formas lingüísticas propuesta por Davydova (2011).

En el contexto de este análisis, ambas propuestas teóricas se han vinculado conceptualmente con las perspectivas objetivas y subjetivas del concepto de complejidad respectivamente. La perspectiva objetiva o absoluta, define el concepto como una propiedad característica del sistema lingüístico, y la perspectiva subjetiva o relativa (user-oriented), explica el concepto desde la perspectiva del costo o dificultad que experimenta el usuario del sistema lingüístico.

\subsubsection{Grado de complejidad del presente perfecto}

En términos objetivos, la naturaleza de la complejidad del presente perfecto se puede explicar en cuanto a:

a) Las relaciones temporales expresadas por la construcción. La evidencia sugiere que la expresión de temporalidad predomina por sobre la expresión de aspectualidad, generando condiciones para el sobreuso del pretérito. Este fenómeno puede ser explicado por la complejidad que reviste el procesamiento de nociones semánticas de temporal recency of the past event, y la dificultad del procesamiento de la construcción como una relación temporal entre un evento en el pasado y su relevancia en el presente, favoreciendo la expresión temporal de un solo evento. 
b) La composición semántica de la forma. Si bien, la construcción en sí no parece implicar un alto grado de complejidad en términos de procesamiento, desde este punto de vista, las nociones semánticas inherentes a los diferentes tipos de situaciones: telicidad, dinamismo y duración, requieren de una capacidad de procesamiento adicional por parte de los individuos para el procesamiento efectivo de esta forma.

c) El carácter polisémico de la forma. Los diferentes usos del presente perfecto: resultativo, experiencial, continuativo y pasado reciente expresan diferentes grados de aspectualidad, y diferentes grados de relación entre las nociones de anterioridad y relevancia. Las distinciones entre estos grados de variación no parecen ser interpretados en el procesamiento de la forma.

d) Secuencia de adquisición. En la secuencia de desarrollo del sistema de interlengua, la adquisición de tiempos simples y la expresión de temporalidad preceden a la adquisición de formas complejas y a la expresión de aspectualidad, generando condiciones para la expresión de temporalidad por sobre aspectualidad por parte de los individuos.

En términos subjetivos, los principios $1 \mathrm{~b}-\mathrm{c}-\mathrm{d}$ formulados por VanPatten (2004) permiten inferir que los alumnos parecen atender al significado expresado por el verbo léxico por sobre el significado expresado por el auxiliar, interpretando de este modo la noción temporal expresada por la frase verbal y no la relación temporal entre las nociones de anterioridad y relevancia.

\subsubsection{Grado de complejidad de la voz pasiva}

En términos objetivos, la complejidad de la voz pasiva se puede explicar en cuanto a:

a) Configuración estructural y semántica de la forma lingüística. En términos estructurales el sujeto de la voz pasiva no expresa agentividad, difiriendo de este modo, con la correspondiente forma activa. La configuración sintáctica de la oración posiciona al objeto directo activo como sujeto pasivo, y le otorga al sujeto activo un carácter opcional en la forma de una frase preposicional opcional. En términos semánticos, la agentividad expresada por esta frase preposicional opcional, adquiere un rol secundario, predominando 
este modo el rol semántico 'afectado' en el sujeto y el rol 'resultativo' de la situación expresada a través de la frase verbal.

En términos subjetivos, el principio de la primera forma nominal explica la tendencia al procesamiento de esta primera forma como sujeto o agente. En términos de procesamiento, los individuos tienden a presentar problemas en el procesamiento de las nociones de transitividad expresada por la frase verbal y los roles semántico asociados a las categorías sintácticas del sujeto y el objeto en una determinada forma.

\subsubsection{Grado de complejidad de las preposiciones}

En términos objetivos, la complejidad de las preposiciones se puede explicar en cuanto a:

a) El carácter polisémico de la forma. De acuerdo a lo establecido anteriormente, un dominio lingüístico en el que se establece una relación directa entre una forma y multiples significados, o viceversa, es más complejo que un dominio en el que esta relación es de uno a uno.

\section{Conclusiones}

El objetivo de nuestro estudio era responder dos preguntas de investigación:

1. ¿Qué estructuras gramaticales del primer año son percibidas como complejas por estudiantes y profesores chilenos del programa de Pedagogía en Inglés en la Universidad de Santiago de Chile?

2. ¿Dónde radica la complejidad de las estructuras identificadas por los alumnos y profesores: ¿en la configuración de la estructura en sí, en el costo de procesamiento de la estructura o la combinación de ambos?

En relación a la primera pregunta investigativa, los resultados permiten concluir que existe un acuerdo general por parte de estos estudiantes y profesores en cuanto a caracterizar las formas perfectas, la voz pasiva y las preposiciones como formas de alta complejidad, principalmente por el esfuerzo cognitivo que demanda la asociación entre estas formas y su(s) significado(s). En este sentido, y entendiendo el procesamiento como un prerrequisito para la adquisición, las expresiones complejas de temporalidad y aspectualidad en conjunto con 
el carácter polisémico propios del presente perfecto, la complejidad de la interpretación de las nociones de agentividad y transitividad propias de la voz pasiva y el carácter polisémico de las preposiciones, parecen constituir las principales características lingüísticas que hacen que estas estructuras sean percibidas como complejas.

Con respecto a la segunda pregunta de investigación, los resultados permiten concluir que no es posible interpretar el grado de complejidad percibido por estudiantes y profesores chilenos de un programa de Pedagogía en Inglés solamente desde una perspectiva objetiva y/o subjetiva. Los diferentes grados de complejidad de las formas anteriormente mencionadas parecen subyacer en la relación entre las representaciones limitadas de los estudiantes por las condiciones de procesamiento propias de un primer año como etapa de aprendizaje.

$\mathrm{Al}$ revisar las características de las estructuras identificadas como complejas por estudiantes y profesores, es posible establecer que la condición de tales encuentra su sustento en las teorías de procesamiento, en los principios de input lingüístico y la noción de complejidad desarrolladas en el marco teórico.

\subsection{Implicaciones pedagógicas y proyecciones}

Los resultados y conclusiones que se derivan de este trabajo suponen una serie de supuestos desde el punto de vista metodológico para los profesores que imparten clases en esta carrera de Pedagogía en Inglés:

1) Las estructuras cognitivamente difíciles requieren un apoyo contextual mediante la personificación del aprendizaje. La complejidad inherente de ciertas estructuras podría bajar y ser más significativa para los estudiantes si las secuencias didácticas proveen un contexto situacional real para la construcción de la representación y, en dicho proceso, los alumnos tienen un rol activo y central.

2) Las secuencias didácticas para trabajar estas estructuras pueden utilizar habilidades que los alumnos ya poseen. Las tareas podrían diseñarse de tal forma que el alumno utilice su conocimiento y habilidades que trae al aula, por ejemplo, como nativo digital, que pueden ayudarle a bajar la complejidad de una estructura.

3) Descongestionar la carga cognitiva de la frase verbal mediante el uso de actividades macro. El uso de metodologías como el aprendizaje por 
proyectos o estudio de casos podría alivianar la carga cognitiva, ya que el foco no es la estructura misma sino un fin mayor que abarca una serie de procesos.

Estas sugerencias pueden ser utilizadas como guía en la elaboración de materiales didácticos, pues se basan en los factores que subyacen la complejidad asociada a determinadas estructuras y abre la posibilidad para continuar explorando el universo de las representaciones que los alumnos tienen y cómo esto influye en su proceso de aprendizaje. Además, se percibe como necesaria la discusión de las implicancias de factores identificados por las propuestas teóricas que dificultan la adquisición de estructuras con cierto nivel de complejidad. Este debate supone la problematización de los procesos cognitivos que ocurren durante el aprendizaje y la comprensión del error en la producción de una L2 como un proceso consustancial al aprendizaje que evidencian que este está ocurriendo.

\section{Referencias bibliográficas}

Abric, J. C. (1976). Jeux, Conflicts et Représentations Sociales. (Tesis doctoral). Université de Provence. Francia.

Buyl, A. \& Housen, A. (2015). Developmental Stages in Receptive Grammar Acquisition: A Processability Theory account. Second Language Research, 31(4), 523-550.

Chaudron, C. (1985). Intake: On methods and models for processing of input. Studies in Second Language Acquisition, 7(1), 1-14.

Cook, G. (2003). Applied Linguistics. Oxford: Oxford University Press.

Council of Europe. (2001). Common European Framework of Reference for Languages: learning, teaching, assessment. New York: Cambridge University Press.

Davydova, J. (2011). The present perfect in non-native Englishes: A corpus based study of variation. Boston: Walter de Grouyer $\mathrm{GmbH} \& \mathrm{Co}$.

Gass, S. M. (1997). Input, interaction, and the second language learner. Mahwah, NJ: Lawrence Erlbaum Associates.

Hall, G. (2011). Exploring English language teaching: Language in action. New York: Routledge. 
Hernández, R., Fernández, C. \& Baptista, P. (2010). Metodología de la investigación. Ciudad de México: McGraw Hill.

Hymes, D. (1972). On communicative competence. En J. B. Pride \& J. Holmes (Eds.), Sociolinguistics (pp. 269-293). Londres: Penguin Books.

Jordan, G. (2004). Theory construction in second language acquisition. Amsterdam/Philadelphia, PA: John Benjamins.

Keatinge, D \& Keßler, J. -U. (2009). The Acquisition of the passive voice in English as a foreign language: Production and perception. En J. -U. Keßler \& D. Keatinge (Eds.), Research in second language acquisition: Empirical evidence across languages (pp. 41-68). Newcastle upon Tyne: Cambridge Scholars.

Kornblit, A. L. (Coord.). (2007). Metodología cualitativas: Modelos y procedimientos de análisis ( $2^{\mathrm{a}}$ ed.). Buenos Aires: Biblos.

Krashen, S. (1981). Second language acquisition and second language learning. New York: Pergamon Press.

Kusters, W. (2003). Linguistic complexity. The influence of social change on verbal inflection. Utrecht: LOT.

Miestamo, M. (2008). Grammatical complexity in a cross-linguistic perspective. En M. Miestamo, K. Sinnemaki, \& F. Karlsson (Eds.), Language complexity. Typology, contact, change (pp. 22-41). Amsterdam/Philadelphia: John Benjamins.

Moscovici, S. (1961). El psicoanálisis, su imagen y su público. Buenos Aires: Huemul.

Pienemann, M. (1998). Language processing and second language development: Processability theory. Amsterdam: John Benjamins. . (2005). An introduction to Processability Theory. En M. Pienemann (Ed.), Crosslinguistic aspects of processability theory (pp. 1-60). Amsterdam/Philadelphia, PA: John Benjamins.

. (2011). Learner variation. En M. Pienemann \& J. U. Keßler (Eds.), Studying processability theory: An introductory textbook (pp. 1226). Amsterdam/Philadelphia, PA: John Benjamins.

Savignon, S. (1983). Communicative competence: Theory and classroom practice; texts and contexts in second language learning. Reading, MA: Addison-Wesley. 
Szmrecsanyi, B. \& Kortmann, B. (2009). Between simplification and complication: Non-standard varieties of English around the world. En G. Sampson, D. Gill, \& P. Trudgill (Eds.), Language complexity as an evolving variable (pp. 64-80). Oxford: Oxford University Press.

VanPatten, B. (2004). Input processing in second language acquisition. En B. VanPatten (Ed.), Processing instruction: Theory, research, and commentary (pp. 5-31). Mahwah, NJ: Lawrence Erlbaum.

Widdowson, H. (1998). Context, Community and Authentic Language. TESOL Quarterly, 32(4), 708-716. 